\title{
Development of a Brushless Motor Driver of Forward and Backward Rotations for UAV
}

\author{
Genki Matsushima, Keichi Yoshino \\ Kitakyushu National College of Technology, 5-20-1 Shii,Kokuraminami-ku, Kitakyushu-shi 802-0985, Japan \\ Corresponding Author: d31036gm@apps.kct.ac.jp
}

\begin{abstract}
In recent years, miniature multi-rotor UAVs have been attracted and developed. These drive motors with the brushless motors are expected to be exploited in various scenes. The purpose of this study is the progress of the position- controlling ability of UAV by the development of the brushless motor driver to enable serial communication with the outside MCU and the brushless motors of forward and backward rotations. This study could actually manufacture the circuit of the brushless motor driver, and trigger the brushless motor.
\end{abstract}

Keywords: Keywords: the brushless motor, the brushless motor driver, PWM of forward and backward.

\section{First}

In recent years, the UAV (Unmanned Air Vehicle) has been prevailed. There are variable kinds of UAV; for instance, the stealth unmanned vehicles of the American military for big ones, the AR. Drone like a small toy showed in Fig. 1, and the spherical ones made by the Japan Defense Agency.

Now, the ways of use these UAVs in Japan are to scatter agricultural chemicals for farmers, and to research in the devastated areas and for processing volcanoes that people cannot enter. From now on, the UAVs are expected to be utilized in more variable scenes and for more variable aims. The big types of UAVs have engines, but the most of small ones drive with electrical motors the most of that are the brushless motors. Though the brushless motor drivers to trigger the brushless motors are on the markets, these are for hobby. Therefore, as these brushless motor drivers communicate slowly and can only forward, so it cannot control the position accurately and complicatedly. To enable controlling position, it needs the brushless motor drivers that can communicate quickly with outside and trigger the brushless motors in forward and backward.

First of all, in this study, after we develop the normal brushless motor driver and enable it to control speed by the serial communication from outside, we enable it forward and backward. In addition, we aim downsizing and lightening it for when it will be equipped with UAVs.

\section{The Feat Of The Brushless Motor}

The brushless motor is generic name of the brushed DC electric motors (hereinafter called DC motor) removed the mechanical parts of contact (commutators, brushes and etc) and equipped with the current switching circuit (hall effect devices, electrical transistor and etc). In brief, it's the motors replaced with the flow straighteners electrically. Generally, the DC motors have some great feats as controlling motors. For example, the radical acceleration, the large starting torque, the goodness of power efficiency, the linear rotation-speed change by the change of the applied voltage, the linearity of the output torque to the incoming current and so on. That is to say this is the great motor with all necessary capabilities for the controlling motor.

While, the fault is that the motor bowl needs a brush and commutator to supply electric power for the motor rotation. Also, this slides at top speed with the motor rotation. For that reason, as the brush and commutator wears and the powder form and carbon spatters, the life of the motor shortens. As it wears more, the spark spatters in the point of contact when it rectifies, and it causes some electrical obstacle like electric corrosion.

In this situation, the motor rectification reasonably gets worse, and it causes some troubles; for instance, the torque occurrence decreases even though excessive current runs. To solve such a problem, the brushless motor without the 
mechanical parts of contact was developed.

As the part of contact is removed from the flow straighteners of DC motors, it brings some kinds of merits. The most one is that it doesn't make noise theoretically for the brushless structure. This means that not only mechanical noise but also electric one doesn't sound. If there are still the part of contact in this motor, it's the shaft bearing. However, this is necessary for even other types of motors, so the life of the brushless motor is not so different to the $\mathrm{AC}$ motor. In other words, as the $\mathrm{DC}$ motor gets converted to the brushless one, the life of the motor lengthens.

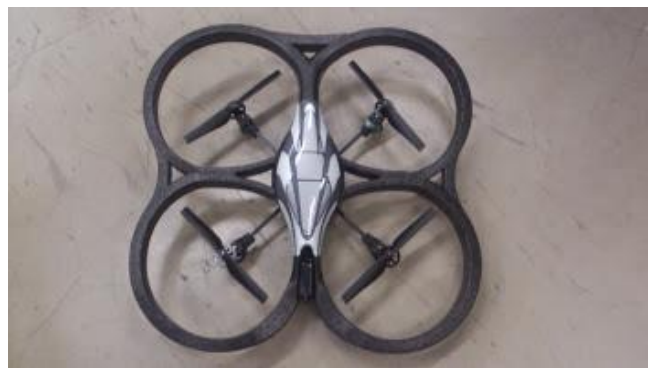

Fig. 1 the example of UAV

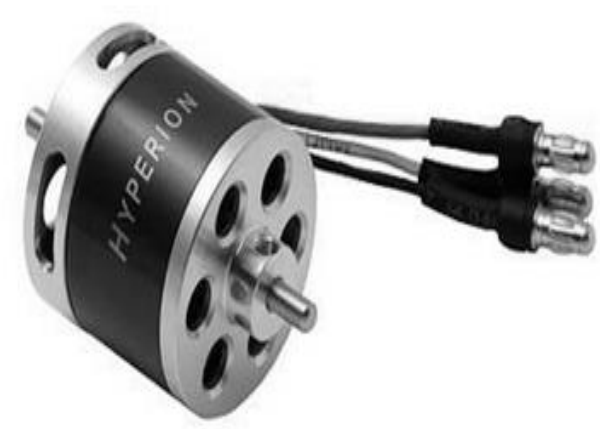

Fig. 2 the example of the brushless motor

Though the above explains that the brushless motor theoretically doesn't make noise for the structure, to be exact, this expression is not necessarily correct. This is because even the brushless motor has to make subtle noise. Certainly, the brushless motor doesn't cause spark like DC motors at the current conversion. However, if the electric current change is abruptly given to inductive load like drive coils, the higher harmonic noise proceeded from the frequency of the rotating magnetic field, and this can badly affects various electronic devices. Also, the very subtle sound noise sounds because of the electric current given to the drive coil and the effect of the magnetic force among rotors. Anyway, the level of noise is very small, and the brushless motor is generally called the noiseless motor. In brief, as the DC motor becomes brushless, any mechanical troubles in commutation mechanism is solved completely. This is the most feat of the brushless motor.

Therefore, the brushless motors are frequently used for UAVs that need the electric motors with high speed rotation and long life.

\section{Classification of Brushless Motor Driver}

\subsection{The classification By Ways to Detect Angle of Rotation}

According to the angle of rotation, the brushless motor driver has to change the voltage toward the terminal at any time. Therefore, it is necessary to detect the angle of rotation of the brushless motor. There are some ways of detection; the magnetic sensor one used by the hall effective devices, and the light sensor one used by the light emitting diode and the photo transistor. In addition, the other one is the sensor less one with electronic-circuit technique. Generally, the rotor of the brushless motor is composed of the permanent magnet. For that reason, as the hall effective devices are used to detect the magnetic pole of the rotor, the angle of rotation can be known from the leakage flux. This means it is unnecessary to make the new especial magnetic field for the magnetic-pole sensor, and this method enables the motor itself become in more simple structure. Also, the hall effective devices are in low prices comparatively. For these reasons, to detect the magnetic field of the brushless motor, the hall effective devices are used.

\subsection{Classification by the Electric Current Supply System}

The brushless motor driver needs that the interchange synchronized with the rotor by the inverter is supplied to the motor. The most basic way to trigger the brushless motor is the six switching element driven by on-off system; the $120^{\circ}$ conduction system and the $180^{\circ}$ conduction system, which are called the short form drive.

The $120^{\circ}$ conduction system is frequently used for the brushless motor. In this controlling method, the switching pattern is decided by the rule; in the six switching element in three phases bridge circuit, the one phase is in the upper row, another one is in the lower row and the other ones are off. The Fig. 3 shows that the arrangement of the electric current as the model of the movement of the six switching element and the magnetic field. In this switching system, the all switch in all phases are on in 120 degrees and off in 60 degrees at the 
power-supply side and the GND side, so this is called the $120^{\circ}$ conduction system. the theoretical voltage waveform between $\mathrm{U}-\mathrm{V}$ of this system is shown in the Fig. 4.

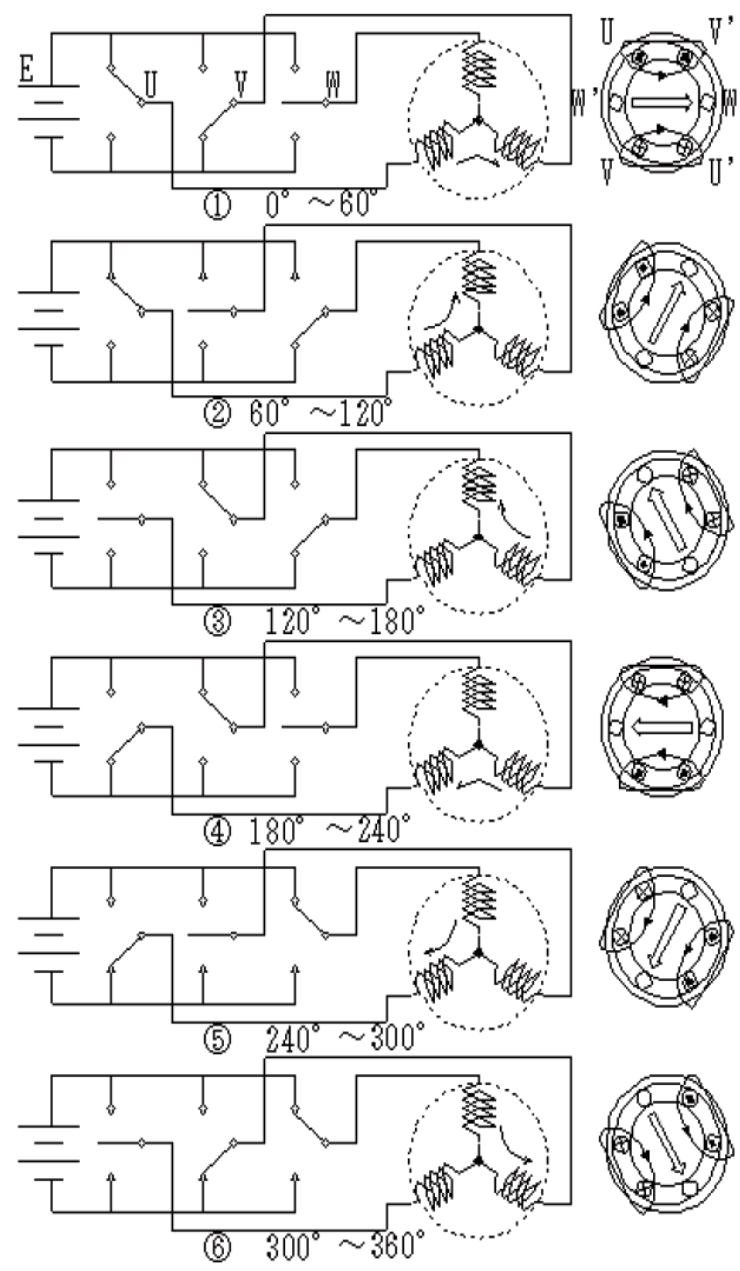

Fig. 3 the switching pattern of the $120^{\circ}$ conduction system.

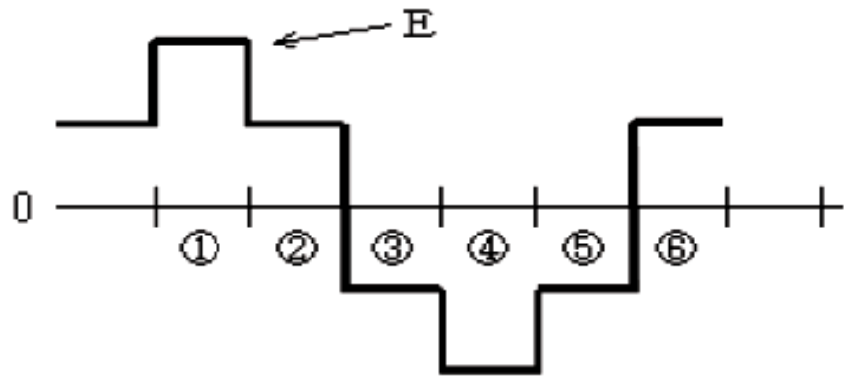

Fig.4 the ideal voltage waveform of the $120^{\circ}$ conduction system.

In the $180^{\circ}$ conduction system, each phase regularly switches the switching element on in the upper row or the lower. The difference between the $120^{\circ}$ conduction system and the $180^{\circ}$ one is that the latter has the possibility that the power supply and GND can short, because there is no time when both the upper row and lower one of switching element are switched off, and both switches are on at the moment when the switch changes from the lower row to the upper. To solve this problem, it needs the time when the switching element in the upper row and the lower shown (dead time) in the Fig. 7.

The Fig. 5 shows the arrangement of the electric current in the $180^{\circ}$ conduction system and the magnetic field.

In this conduction system, it repeats that each phase turns on electricity at the $180^{\circ}$ in the lower row after it does in the upper. Therefore it is called the $180^{\circ}$ conduction system. The theoretical voltage waveform between $\mathrm{U}-\mathrm{V}$ of this system is shown in the Fig. 6.

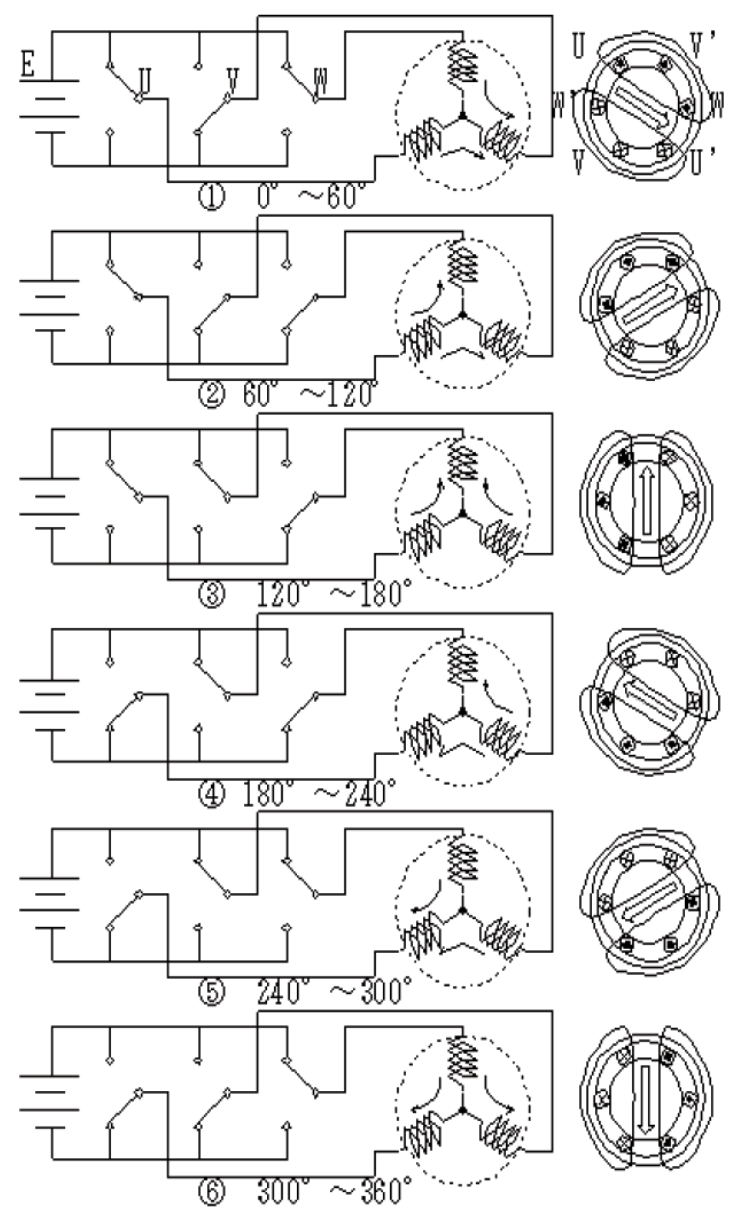

Fig.5 the switching pattern of the $180^{\circ}$ conduction system.

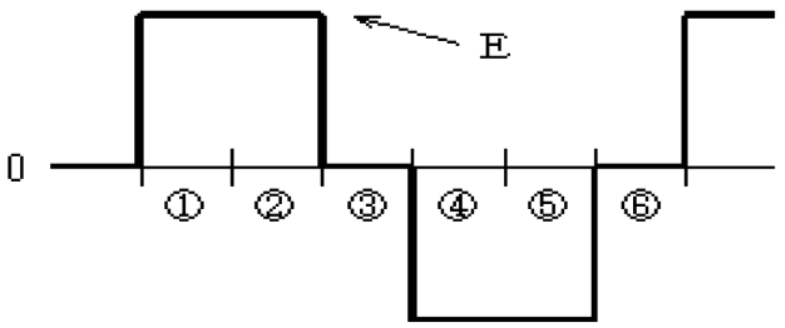

Fig. 6 the ideal voltage waveform of the $180^{\circ}$ conduction system. 


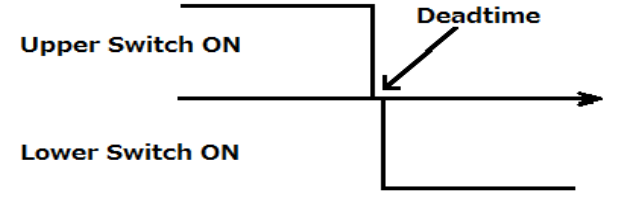

Fig. 7 the insertion of the dead time

The $180^{\circ}$ conduction system has the good point that its control is easy and the all-purpose ICs are sold in markets, but it can easily cause noise and oscillation, and it's worse than the $180^{\circ}$ one efficiently. While, the $180^{\circ}$ one is more efficient than the $120^{\circ}$ one and doesn't make much noise and oscillation, but it's vulnerable to the excessive load change, cannot be controlled when the position moved and is more complex to control than the $120^{\circ}$ one.

\section{The Structure of the System Surrounding the Brushless Motor Driver}

The Fig. 8 shows the structure of the system in the brushless motor, the brushless motor driver and the outside MCU. This Fig. is for the sensor less type of the brushless motor driver produced this time. The sensing circuit is to detect the angle of rotation in the brushless motor. As it detects, it sends the signal with the information about the angle of rotation toward the inside MCU. When the inside MCU receives the signal from the sensing circuit, it sends six signals to the switching circuit with following by the switching pattern of the conduction system to revolt the brushless motor at the speed set in advance. The switching circuit makes three phases from the six signals and one phase from the two signals, and sends it to the brushless motor. In this way, the brushless motor driver outputs the three phase inputs of the brushless motor. In the case of the speed change, it can be done by the outside MCU sends the signal of speed to the inside one.

\section{Motor Driver}

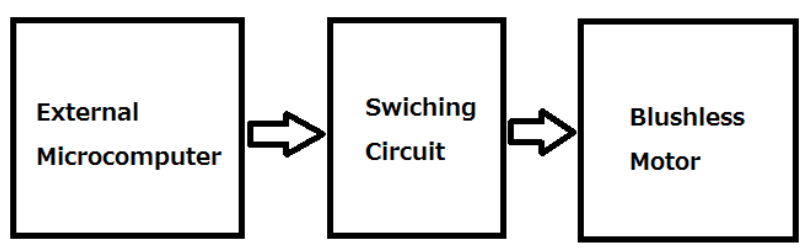

Fig. 8 the Structure of the System Surrounding the Brushless Motor Driver

\section{Forward Reverse Control}

Normally, the brushless motor drivers sold in markets can only forward the brushless motors. This is because these drivers for hobby, that are used in the radio-controlled helicopter, so it's enough to forward only. However, for the UAVs controlling the plural brushless motor, to enable backward can improve the controlling ability. Therefore, this study aims to produce the brushless motor driver enabling forward and backward.

\section{The Switching Circuit}

The Fig.9 shows the diagram of the switching circuit made this time. As the structure of the system surrounding the brushless motor driver is explained at 3rd page, the switching circuit makes three phases by the six signals from the inside MCU of the brushless motor driver. Therefore, actually three circuits were made in the Fig.9.

\section{The Motion Experiment of the Brushless Motor Driver}

\subsection{Purpose}

We test whether the brushless motor rotates or not with the switching circuit on the universal base. This experiment is to confirm the movement of the switching circuit, so it doesn't research about the detecting the angle of rotation of the brushless motor by the sensing circuit.

\subsection{Preparation}

The Fig. 10 shows the circuit of the brushless motor driver made this time. The Arduino UNO is used as the inside MCU. The switching pattern of the $120^{\circ}$ conduction system shown in the Fig. 3 is inputted into the circuit produced from this MCU on 12[ms] cycle regardless of the angle of rotation of the brushless motor. VCC, the power supply of the circuit, is set in 10[V].

In addition, the Fig. 10 includes HP ZS2213-22 of HYPERION used in this experimental. The Normal rated power of HP ZS2213-22 is set in 80[W].

\subsection{Result}

As the power supply applies the circuit, the brushless motor rotates or doesn't rotate. In the case of that it doesn't rotate, it starts to rotate with the initial response by hand. The brushless motor backwards, when the opposite order of the switching pattern of the $120^{\circ}$ conduction system inputs the switching circuit.

The Fig. 11 shows the voltage waveform measured in 
this experiment. The peak of the voltage waveform is about $8[\mathrm{~V}]$, the least is about $12[\mathrm{~V}]$, and the cycle is $12[\mathrm{~ms}](=83[\mathrm{HZ}])$. The electric-current $12[\mathrm{~A}]$ runs in the circuit.

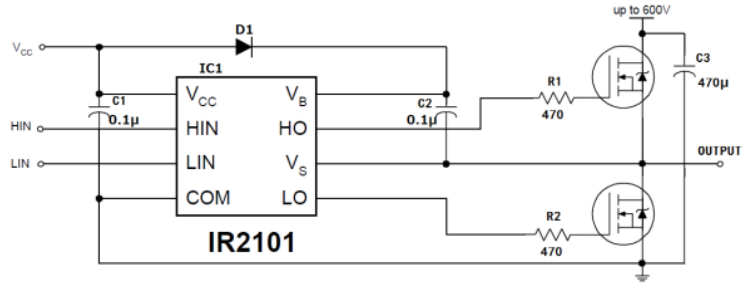

Fig. 9 the part of the circuit diagram

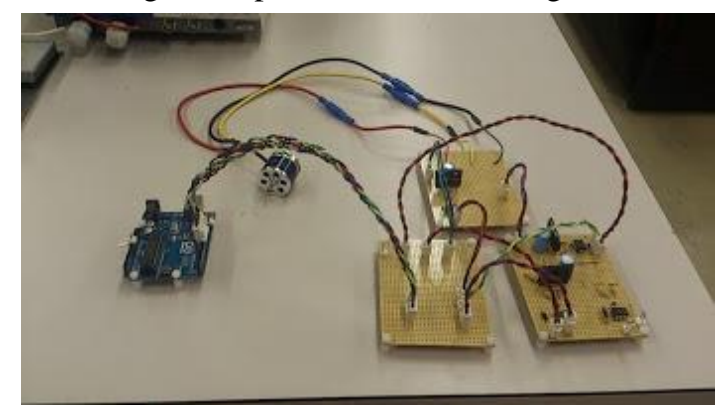

Fig. 10 the circuit of the brushless motor driver

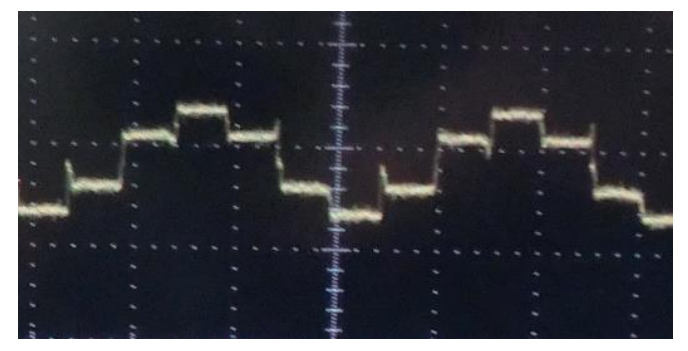

Fig. 11 the voltage waveform measured

\subsection{Examination}

As the peak, the least and the cycle of the voltage waveform measured compare, these are equal almost. Therefore, it indicates that the voltage waveform measured was same as the theoretical one.

When the power supply applies the circuit, the brushless motor didn't rotate sometimes. The reason is that the brushless motor may step out. It may be that the angle of rotation of the brushless motor couldn't be detected and it stepped out because the sensing circuit was not implemented. The resistance component of the brushless motor might become bigger as the rotation speed of the brushless motor got faster, become smaller as it got slower. Therefore, the electric-current in 12[A] in the circuit might decrease as the rotation speed of the brushless motor got faster, increase as it got slower. The NRP of the brushless motor in this experiment was $80[\mathrm{~W}]$, so it operated in less than the NRP. However, the counter electromotive force of the brushless motor became small when the rotation speed got slow, so the electricity consumption of the brushless motor can rise and exceed the NRP. Therefore, it needs the provision like that the applied voltage lowers at the beginning.

Also, it needs counterseals against the possibility of that the elements like MOS-FET can be broken as the big electric-current runs in the switching circuit.

To backward, the order of the switching pattern, that inputs from the inside MCU to the switching circuit, in the Fig. 3 has to be arranged in opposite.

\section{Conclusion}

Though we designed the circuit of the brushless motor driver, we couldn't make the brushless motor rotate at first. However, it succeeded that the brushless motor could rotate by repeating the experiments and fixing the circuit. In the case of that it didn't rotate, it could do with the initial response by hand.

\section{Subject in Future}

The brushless motor could be rotated with the circuit we made, but it's necessary to produce the sensing circuit, that detects the angle of rotation of the brushless motor, to prevent the stepping out at the beginning. In addition, the brushless motor speeds up gradually, and prevent to step out. Also, to prevent the breaking elements if the big current runs in the switching circuit, we connect some MOS-FETs in parallel. When the backward from the forwarding, it needs provision against stepping out.

\section{Acknowledgement}

I thanks too much to Mr. Yoshino for your instruction in this study and to members of Yoshino laboratory for you information and advice about the design of the circuit.

\section{References}

(1) Denpa Shinbunsya :"The practical technique of the brushless motor", pp. 2-11, 32-68, Radio News Service, 2005 